\title{
The exponential stability of BAM neural networks with leakage time-varying delays and sampled-data state feedback input
}

\author{
Li Li ${ }^{1}, 2$, Yongqing Yang ${ }^{1 *}$, Tian Liang ${ }^{1}$ and Manfeng $\mathrm{Hu}^{1}$
}

${ }^{*}$ Correspondence:

yyq640613@gmail.com

${ }^{1}$ School of Science, Jiangnan

University, Wuxi, 214122, China

Full list of author information is

available at the end of the article

\begin{abstract}
In this paper, the exponential stability of bidirectional associative memory neural networks with leakage time-varying delays and sampled-data state feedback input is considered. By applying the time-delay approach, some conditions for ensuring the stability of a system are obtained. In addition, a numerical example is given to demonstrate the effectiveness of the obtained results.
\end{abstract}

Keywords: BAM neural networks; leakage delays; sampled data

\section{Introduction}

In the past few decades, neural networks have been widely investigated by researchers. In 1987, bidirectional associative memory (BAM) neural networks were firstly introduced by Kosko $[1,2]$. Due to its better abilities as information associative memory, the BAM neural network has attracted considerable attention in different fields such as signal processing, pattern recognition, optimization, and so on.

It is well known that time delay is unavoidable in the hardware implementation of neural networks due to the finite switching speed of neurons and amplifiers. The delay can cause instability, oscillation, or poor dynamical behavior. In practical applications, there exist many types of time delays such as discrete delays [3], time-varying delays [4], distributed delays [5, 6], random delays [7] and leakage delays (or forgetting delays) [8, 9]. Up to now, a large number of results about delay BAM neural networks have been reported [10-13]. All of the conclusions could be roughly summarized into two types: in terms of the stability analysis of equilibrium points, and of the existence and stability of periodic or almost periodic solutions.

The leakage delay, which exists in the negative feedback term of a neural network system, emerges as a research topic of primary importance recently. Gopalsamy [8] investigated the stability of the BAM neural networks with constant leakage delays. Further, Liu [14] discussed the global exponential stability for BAM neural networks with timevarying leakage delays, which extend and improve the main results of Gopalsamy. Peng et al. [15-17] derived the stability criteria for the BAM neural networks with leakage delays, unbounded distributed delays and probabilistic time-varying delays.

Sampled-data state feedback is a practical and useful control scheme and has been studied extensively over the past decades. There are some results dealing with synchronization $[18,19]$, state estimate [20-22] and stability [23-29]. Recently, the work in [24] has 
studied the problem of the stability of sampled-data piecewise affine systems via the input delay approach. Although the importance of the stability of neural networks has been widely recognized, no related results have been established for the sampled-data stability of BAM neural networks with leakage time-varying delays. Motivated by the works above, we consider the sampled-data stability of BAM neural networks with leakage time-varying delays under variable sampling with a known upper bound on the sampling intervals.

The organization of this paper is as follows. In Section 2, the problem is formulated and some basic preliminaries and assumptions are given. The main results are presented in Section 3. In Section 4, a numerical example is given to demonstrate the effectiveness of the obtained results. Some conclusions are proposed in Section 5.

\section{Preliminaries}

In this paper, we consider the following BAM neural networks with leakage time-varying delays and sampled-data state feedback inputs:

$$
\left\{\begin{array}{l}
\dot{x}_{i}(t)=-a_{i} x_{i}\left(t-\rho_{i}(t)\right)+\sum_{j=1}^{n} b_{i j}^{(1)} g_{j}\left(y_{j}(t)\right)+\sum_{j=1}^{n} b_{i j}^{(2)} g_{j}\left(y_{j}\left(t-\tau_{i j}(t)\right)\right)+\tilde{u}_{i}(t), \\
\dot{y}_{i}(t)=-c_{i} y_{i}\left(t-r_{i}(t)\right)+\sum_{j=1}^{n} d_{i j}^{(1)} f_{j}\left(x_{j}(t)\right)+\sum_{j=1}^{n} d_{i j}^{(2)} f_{j}\left(x_{j}\left(t-\sigma_{i j}(t)\right)\right)+\tilde{v}_{i}(t),
\end{array}\right.
$$

where $i \in \tilde{N}=\{1,2, \ldots, n\}$, the $x_{i}(t)$ and $y_{i}(t)$ are neuron state variables, the positive constants $a_{i}$ and $c_{i}$ denote the time scales of the respective layers of the networks, $b_{i j}^{(1)}, b_{i j}^{(2)}$, $d_{i j}^{(1)}, d_{i j}^{(2)}$ are connection weights of the network. $\rho_{i}(t)$ and $r_{i}(t)$ denote the leakage delays, $\tau_{i j}(t)$ and $\sigma_{i j}(t)$ are time-varying delays, $f_{j}(\cdot), g_{j}(\cdot)$ are neuron activation functions, $\tilde{u}_{i}(t)=-k_{i} x_{i}\left(t_{k}\right), \tilde{v}_{i}(t)=-l_{i} y_{i}\left(t_{k}\right)$ are sampled-data state feedback inputs, $t_{k}$ denotes the sample time point, $t_{k} \leq t<t_{k+1}, k \in \mathbb{N}, \mathbb{N}$ denotes the set of all natural numbers.

Assume that there exists a positive constant $L$ such that the sample interval $t_{k+1}-t_{k} \leq L$, $k \in \mathbb{N}$. Let $d_{k}(t)=t-t_{k}$, for $t \in\left[t_{k}, t_{k+1}\right)$, then $t_{k}=t-d_{k}(t)$ with $0 \leq d_{k}(t) \leq L$.

For the sake of convenience, we give the following notations:

$$
\begin{array}{ll}
\bar{\rho}_{i}=\sup _{t \in R} \rho_{i}(t), & \underline{\rho_{i}}=\inf _{t \in R} \rho_{i}(t), \\
\bar{r}_{i}=\sup _{t \in R} r_{i}(t), & \underline{r_{i}}=\inf _{t \in R} r_{i}(t), \\
\bar{\tau}_{i j}=\sup _{t \in R} \tau_{i j}(t), & \underline{\tau_{i j}}=\inf _{t \in R} \tau_{i j}(t), \\
\bar{\sigma}_{i j}=\sup _{t \in R} \sigma_{i j}(t), & \underline{\sigma_{i j}}=\inf _{t \in R} \sigma_{i j}(t), \\
\rho^{*}=\sup _{t \in R} \dot{\rho}_{i}(t), & r^{*}=\sup _{t \in R} \dot{r}_{i}(t) .
\end{array}
$$

Before ending this section, we introduce two assumptions, which will be used in next section.

Assumption 1 There exist constants $L_{j}^{f}>0, L_{j}^{g}>0$ such that

$$
0 \leq \frac{f_{j}(x)-f_{j}(y)}{x-y} \leq L_{j}^{f}, \quad 0 \leq \frac{g_{j}(x)-g_{j}(y)}{x-y} \leq L_{j}^{g},
$$

for all $x, y \in \mathbb{R}, x \neq y$ and $j \in \tilde{N}$. 
Assumption 2 Let $a_{i} \bar{\rho}_{i}<1, c_{i} \bar{r}_{i}<1$, for all $i \in \tilde{N}$. There exist positive constants $\xi_{1}, \xi_{2}, \ldots, \xi_{n}$ and $\eta_{1}, \eta_{2}, \ldots, \eta_{n}$ such that, for $t>0$ and $i \in \widetilde{N}$, the following inequalities hold:

$$
\left\{\begin{array}{l}
-\left[a_{i}\left(1-2 a_{i} \bar{\rho}_{i}\right)-a_{i} \rho^{*}-k_{i}\right] \frac{1}{1-a_{i} \bar{\rho}_{i}} \xi_{i}+\left[\sum_{j=1}^{n}\left|b_{i j}^{(1)}\right|+\sum_{j=1}^{n}\left|b_{i j}^{(2)}\right|\right] L_{j}^{g} \frac{1}{1-c_{j} \bar{r}_{j}} \eta_{j}<0, \\
-\left[c_{i}\left(1-2 c_{i} \bar{r}_{i}\right)-c_{i} r^{*}-l_{i}\right] \frac{1}{1-c_{i} \bar{r}_{i}} \eta_{i}+\left[\sum_{j=1}^{n}\left|d_{i j}^{(1)}\right|+\sum_{j=1}^{n}\left|d_{i j}^{(2)}\right|\right] L_{j}^{f} \frac{1}{1-a_{j} \bar{\rho}_{j}} \xi_{j}<0 .
\end{array}\right.
$$

\section{Main results}

In this section, we investigate the exponential stability of (1). By using the input delay approach [24], (1) can be rewritten in the following form:

$$
\left\{\begin{aligned}
\dot{x}_{i}(t)= & -a_{i} x_{i}\left(t-\rho_{i}(t)\right)+\sum_{j=1}^{n} b_{i j}^{(1)} g_{j}\left(y_{j}(t)\right) \\
& +\sum_{j=1}^{n} b_{i j}^{(2)} g_{j}\left(y_{j}\left(t-\tau_{i j}(t)\right)\right)-k_{i} x_{i}\left(t-d_{k}(t)\right) \\
\dot{y}_{i}(t)= & -c_{i} y_{i}\left(t-r_{i}(t)\right)+\sum_{j=1}^{n} d_{i j}^{(1)} f_{j}\left(x_{j}(t)\right) \\
& +\sum_{j=1}^{n} d_{i j}^{(2)} f_{j}\left(x_{j}\left(t-\sigma_{i j}(t)\right)\right)-l_{i} y_{i}\left(t-d_{k}(t)\right)
\end{aligned}\right.
$$

The initial conditions of (3) are: $x_{i}(s)=\phi_{i}(s), y_{i}(s)=\varphi_{i}(s), s \in(-\infty, 0], i \in \tilde{N}$, where $\phi_{i}(s)$ and $\varphi_{i}(s)$ are continuous functions on $(-\infty, 0]$.

The main results are stated as follows.

Theorem 1 Let Assumptions 1 and 2 hold; then the BAM neural network (3) is exponentiallystable, i.e., there exists a positive constant $\lambda$ such that $\left|x_{i}(t)\right|=O\left(e^{-\lambda t}\right),\left|y_{i}(t)\right|=O\left(e^{-\lambda t}\right)$, $i \in \widetilde{N}$.

Proof Define the continuous functions

$$
\left\{\begin{aligned}
\Phi_{i}(\omega)= & -\left[\left(a_{i}-\omega\right)\left(1-2 a_{i} \bar{\rho}_{i}\right)-a_{i}\left(e^{\omega \bar{\rho}_{i}}-\left(1-\rho^{*}\right)-k_{i} e^{\omega L}\right)\right] \frac{1}{1-a_{i} \bar{\rho}_{i}} \xi_{i} \\
& +\left[\sum_{j=1}^{n}\left|b_{i j}^{(1)}\right|+e^{\omega \bar{\tau}_{i j}} \sum_{j=1}^{n}\left|b_{i j}^{(2)}\right|\right] L_{j}^{g} \frac{1}{1-c_{j} \bar{r}_{j}} \eta_{j} \\
\Phi_{n+i}(\omega)= & -\left[\left(c_{i}-\omega\right)\left(1-2 c_{i} \bar{r}_{i}\right)-c_{i}\left(e^{\omega \bar{r}_{i}}-\left(1-r^{*}\right)-l_{i} e^{\omega L}\right)\right] \frac{1}{1-c_{i} \bar{r}_{i}} \eta_{i} \\
& +\left[\sum_{j=1}^{n}\left|d_{i j}^{(1)}\right|+e^{\omega \bar{\sigma}_{i j}} \sum_{j=1}^{n}\left|d_{i j}^{(2)}\right|\right] L_{j}^{f} \frac{1}{1-a_{j} \bar{\rho}_{j}} \xi_{j},
\end{aligned}\right.
$$

where $\omega \geq 0, i \in \tilde{N}$.

By Assumption 2, we have

$$
\left\{\begin{aligned}
\Phi_{i}(0)= & -\left[a_{i}\left(1-2 a_{i} \bar{\rho}_{i}\right)-a_{i} \rho^{*}-k_{i}\right] \frac{1}{1-a_{i} \bar{\rho}_{i}} \xi_{i}+\left[\sum_{j=1}^{n}\left|b_{i j}^{(1)}\right|+\sum_{j=1}^{n}\left|b_{i j}^{(2)}\right|\right] \\
& \times L_{j}^{g} \frac{1}{1-c_{j} \bar{r}_{j}} \eta_{j}<0 \\
\Phi_{n+i}(0)= & -\left[c_{i}\left(1-2 c_{i} \bar{r}_{i}\right)-c_{i} r^{*}-l_{i}\right] \frac{1}{1-c_{i} \bar{r}_{i}} \eta_{i}+\left[\sum_{j=1}^{n}\left|d_{i j}^{(1)}\right|+\sum_{j=1}^{n}\left|d_{i j}^{(2)}\right|\right] \\
& \times L_{j}^{f} \frac{1}{1-a_{j} \bar{\rho}_{j}} \xi_{j}<0 .
\end{aligned}\right.
$$

Because $\Phi_{i}(\omega)$ and $\Phi_{n+i}(\omega)$ are continuous functions, we can choose a small positive constant $\lambda$ such that, for all $i \in \tilde{N}$,

$$
\left\{\begin{aligned}
\Phi_{i}(\lambda)= & -\left[\left(a_{i}-\lambda\right)\left(1-2 a_{i} \bar{\rho}_{i}\right)-a_{i}\left[e^{\lambda \bar{\rho}_{i}}-\left(1-\rho^{*}\right)\right]-k_{i} e^{\lambda L}\right] \frac{1}{1-a_{i} \bar{\rho}_{i}} \xi_{i} \\
+ & {\left[\sum_{j=1}^{n}\left|b_{i j}^{(1)}\right|+e^{\lambda \bar{\tau}_{i j}} \sum_{j=1}^{n}\left|b_{i j}^{(2)}\right|\right] L_{j}^{g} \frac{1}{1-c_{j} \bar{r}_{j}} \eta_{j}<0, } \\
\Phi_{n+i}(\lambda)= & -\left[\left(c_{i}-\lambda\right)\left(1-2 c_{i} \bar{r}_{i}\right)-c_{i}\left[e^{\lambda \bar{r}_{i}}-\left(1-r^{*}\right)\right]-l_{i} e^{\lambda L}\right] \frac{1}{1-c_{i} \bar{r}_{i}} \eta_{i} \\
& +\left[\sum_{j=1}^{n}\left|d_{i j}^{(1)}\right|+e^{\lambda \bar{\sigma}_{i j}} \sum_{j=1}^{n}\left|d_{i j}^{(2)}\right|\right] L_{j}^{f} \frac{1}{1-a_{j} \bar{\rho}_{j}} \xi_{j}<0 .
\end{aligned}\right.
$$


Let

$$
X_{i}(t)=e^{\lambda t} x_{i}(t)-\int_{t-\rho_{i}(t)}^{t} a_{i} e^{\lambda s} x_{i}(s) d s, \quad Y_{i}(t)=e^{\lambda t} y_{i}(t)-\int_{t-r_{i}(t)}^{t} c_{i} e^{\lambda s} y_{i}(s) d s, \quad i \in \tilde{N}
$$

Calculating the derivative of $X_{i}$ and $Y_{i}$ along the solution of (3), we have

$$
\begin{aligned}
\dot{X}_{i}(t)= & \lambda e^{\lambda t} x_{i}(t)+e^{\lambda t} \dot{x}_{i}(t)-a_{i}\left[e^{\lambda t} x_{i}(t)-\left(1-\dot{\rho}_{i}(t)\right) e^{\lambda\left(t-\rho_{i}(t)\right)} x_{i}\left(t-\rho_{i}(t)\right)\right] \\
= & \lambda e^{\lambda t} x_{i}(t)+e^{\lambda t}\left[-a_{i} x_{i}\left(t-\rho_{i}(t)\right)+\sum_{j=1}^{n} b_{i j}^{(1)} g_{j}\left(y_{j}(t)\right)+\sum_{j=1}^{n} b_{i j}^{(2)} g_{j}\left(y_{j}\left(t-\tau_{i j}(t)\right)\right)\right. \\
& \left.-k_{i} x_{i}\left(t-d_{k}(t)\right)\right]-a_{i} e^{\lambda t} x_{i}(t)+a_{i}\left(1-\dot{\rho}_{i}(t)\right) e^{\lambda\left(t-\rho_{i}(t)\right)} x_{i}\left(t-\rho_{i}(t)\right) \\
= & \lambda e^{\lambda t} x_{i}(t)-a_{i} e^{\lambda t} x_{i}(t)+a_{i}\left(1-\dot{\rho}_{i}(t)\right) e^{\lambda\left(t-\rho_{i}(t)\right)} x_{i}\left(t-\rho_{i}(t)\right) \\
& -a_{i} e^{\lambda t} x_{i}\left(t-\rho_{i}(t)\right)-k_{i} e^{\lambda t} x_{i}\left(t-d_{k}(t)\right) \\
& +e^{\lambda t}\left[\sum_{j=1}^{n} b_{i j}^{(1)} g_{j}\left(y_{j}(t)\right)+\sum_{j=1}^{n} b_{i j}^{(2)} g_{j}\left(y_{j}\left(t-\tau_{i j}(t)\right)\right)\right] \\
= & -\left(a_{i}-\lambda\right) X_{i}(t)-\left(a_{i}-\lambda\right) \int_{t-\rho_{i}(t)}^{t} a_{i} e^{\lambda s} x_{i}(s) d s \\
& -\left[a_{i}-a_{i}\left(1-\dot{\rho}_{i}(t)\right) e^{-\lambda \rho_{i}(t)}\right] e^{\lambda t} x_{i}\left(t-\rho_{i}(t)\right)-k_{i} e^{\lambda t} x_{i}\left(t-d_{k}(t)\right) \\
& +e^{\lambda t}\left[\sum_{j=1}^{n} b_{i j}^{(1)} g_{j}\left(y_{j}(t)\right)+\sum_{j=1}^{n} b_{i j}^{(2)} g_{j}\left(y_{j}\left(t-\tau_{i j}(t)\right)\right)\right]
\end{aligned}
$$

and

$$
\begin{aligned}
\dot{Y}_{i}(t)= & \lambda e^{\lambda t} y_{i}(t)+e^{\lambda t} \dot{y}_{i}(t)-c_{i}\left[e^{\lambda t} y_{i}(t)-\left(1-\dot{r}_{i}(t)\right) e^{\lambda\left(t-r_{i}(t)\right)} y_{i}\left(t-r_{i}(t)\right)\right] \\
= & \lambda e^{\lambda t} y_{i}(t)+e^{\lambda t}\left[-c_{i} y_{i}\left(t-r_{i}(t)\right)+\sum_{j=1}^{n} d_{i j}^{(1)} f_{j}\left(x_{j}(t)\right)+\sum_{j=1}^{n} d_{i j}^{(2)} f_{j}\left(x_{j}\left(t-\sigma_{i j}(t)\right)\right)\right. \\
& \left.-l_{i} y_{i}\left(t-d_{k}(t)\right)\right]-c_{i}\left[e^{\lambda t} y_{i}(t)-\left(1-\dot{r}_{i}(t)\right) e^{\lambda\left(t-r_{i}(t)\right)} y_{i}\left(t-r_{i}(t)\right)\right] \\
= & -\left(c_{i}-\lambda\right) Y_{i}(t)-\left(c_{i}-\lambda\right) \int_{t-r_{i}(t)}^{t} c_{i} e^{\lambda s} y_{i}(s) d s \\
& -\left[c_{i}-c_{i}\left(1-\dot{r}_{i}(t)\right) e^{-\lambda r_{i}(t)}\right] e^{\lambda t} y_{i}\left(t-r_{i}(t)\right)-l_{i} e^{\lambda t} y_{i}\left(t-d_{k}(t)\right) \\
& +e^{\lambda t}\left[\sum_{j=1}^{n} d_{i j}^{(1)} f_{j}\left(x_{j}(t)\right)+\sum_{j=1}^{n} d_{i j}^{(2)} f_{j}\left(x_{j}\left(t-\sigma_{i j}(t)\right)\right)\right] .
\end{aligned}
$$

We define a positive constant $M$ as follows:

$$
M=\max _{1 \leq i \leq n}\left\{\sup _{t \in(-\infty, 0]}\left|X_{i}(t)\right|, \sup _{t \in(-\infty, 0]}\left|Y_{i}(t)\right|\right\}, \quad M>0 .
$$


Let $K$ be a positive number such that

$$
\left\{\begin{array}{l}
\left|X_{i}(t)\right| \leq M<K \xi_{i}, \\
\left|Y_{i}(t)\right| \leq M<K \eta_{i},
\end{array} \quad \text { for all } t \in(-\infty, 0] .\right.
$$

Now, we will prove that

$$
\left\{\begin{array}{l}
\left|X_{i}(t)\right| \leq M<K \xi_{i}, \quad \text { for all } t>0 . \\
\left|Y_{i}(t)\right| \leq M<K \eta_{i},
\end{array}\right.
$$

Let $t_{0}=0$, we firstly prove

$$
\left\{\begin{array}{l}
\left|X_{i}(t)\right| \leq M<K \xi_{i}, \\
\left|Y_{i}(t)\right| \leq M<K \eta_{i},
\end{array} \quad \text { for } t \in\left[t_{0}, t_{1}\right) .\right.
$$

In fact, if it is not valid, there exist $i \in \tilde{N}, t_{0}^{*} \in\left[t_{0}, t_{1}\right)$ such that at least one of the following cases occurs:

$$
\left\{\begin{array}{rlrl}
\text { (a) } X_{i}\left(t_{0}^{*}\right)=K \xi_{i}, \quad \dot{X}_{i}\left(t_{0}^{*}\right) \geq 0, & \left|X_{j}(t)\right|<K \xi_{j}, & & \left|Y_{j}(t)\right|<K \eta_{j}, \\
\text { for } t \in\left(-\infty, t_{0}^{*}\right), j \in \widetilde{N}, & & \\
\text { (b) } X_{i}\left(t_{0}^{*}\right)=-K \xi_{i}, \quad \dot{X}_{i}\left(t_{0}^{*}\right) \leq 0, & \left|X_{j}(t)\right|<K \xi_{j}, & & \left|Y_{j}(t)\right|<K \eta_{j}, \\
& \text { for } t \in\left(-\infty, t_{0}^{*}\right), j \in \widetilde{N}, & & \\
\text { (c) } Y_{i}\left(t_{0}^{*}\right)=K \eta_{i}, \quad \dot{Y}_{i}\left(t_{0}^{*}\right) \geq 0, & \left|X_{j}(t)\right|<K \xi_{j}, & & \left|Y_{j}(t)\right|<K \eta_{j}, \\
& \text { for } t \in\left(-\infty, t_{0}^{*}\right), j \in \widetilde{N}, & & \\
\text { (d) } Y_{i}\left(t_{0}^{*}\right)=-K \eta_{i}, \quad \dot{Y}_{i}\left(t_{0}^{*}\right) \leq 0, & \left|X_{j}(t)\right|<K \xi_{j}, & & \left|Y_{j}(t)\right|<K \eta_{j}, \\
\text { for } t \in\left(-\infty, t_{0}^{*}\right), j \in \widetilde{N} . & &
\end{array}\right.
$$

For $t \in\left(-\infty, t_{0}^{*}\right], j \in \widetilde{N}$,

$$
\begin{aligned}
e^{\lambda t}\left|x_{j}(t)\right| & \leq\left|e^{\lambda t} x_{j}(t)-\int_{t-\rho_{j}(t)}^{t} a_{j} e^{\lambda s} x_{j}(s) d s\right|+\left|\int_{t-\rho_{j}(t)}^{t} a_{j} e^{\lambda s} x_{j}(s) d s\right| \\
& \leq K \xi_{j}+a_{j} \bar{\rho}_{j} \sup _{s \in\left(-\infty, t_{0}^{*}\right]} e^{\lambda s}\left|x_{j}(s)\right| .
\end{aligned}
$$

Hence

$$
e^{\lambda t}\left|x_{j}(t)\right| \leq \sup _{s \in\left(-\infty, t_{0}^{*}\right]} e^{\lambda s}\left|x_{j}(s)\right| \leq \frac{K \xi_{j}}{1-a_{j} \bar{\rho}_{j}} .
$$

Similarly, we have

$$
e^{\lambda t}\left|y_{j}(t)\right| \leq \sup _{s \in\left(-\infty, t_{0}^{*}\right]} e^{\lambda s}\left|y_{j}(s)\right| \leq \frac{K \eta_{j}}{1-c_{j} \bar{r}_{j}} .
$$

If (a) holds, we get

$$
\begin{aligned}
\dot{X}_{i}\left(t_{0}^{*}\right)= & -\left(a_{i}-\lambda\right) X_{i}\left(t_{0}^{*}\right)-\left(a_{i}-\lambda\right) \int_{t_{0}^{*}-\rho_{i}\left(t_{0}^{*}\right)}^{t_{0}^{*}} a_{i} e^{\lambda s} x_{i}(s) d s \\
& -\left[a_{i}-a_{i}\left(1-\dot{\rho}_{i}\left(t_{0}^{*}\right)\right) e^{-\lambda \rho_{i}\left(t_{0}^{*}\right)}\right] e^{\lambda t_{0}^{*}} x_{i}\left(t_{0}^{*}-\rho_{i}\left(t_{0}^{*}\right)\right)-k_{i} e^{\lambda t_{0}^{*}} x_{i}\left(t_{0}^{*}-d_{k}\left(t_{0}^{*}\right)\right)
\end{aligned}
$$




$$
\begin{aligned}
& +e^{\lambda t_{0}^{*}}\left[\sum_{j=1}^{n} b_{i j}^{(1)} g_{j}\left(y_{j}\left(t_{0}^{*}\right)\right)+\sum_{j=1}^{n} b_{i j}^{(2)} g_{j}\left(y_{j}\left(t_{0}^{*}-\tau_{i j}\left(t_{0}^{*}\right)\right)\right)\right] \\
& \leq-\left(a_{i}-\lambda\right) K \xi_{i}+\left(a_{i}-\lambda\right) a_{i} \bar{\rho}_{i} \frac{K \xi_{i}}{1-a_{i} \bar{\rho}_{i}} \\
& +\left[a_{i}-a_{i}\left(1-\dot{\rho}_{i}\left(t_{0}^{*}\right)\right) e^{-\lambda \rho_{i}\left(t_{0}^{*}\right)}\right] e^{\lambda \rho_{i}\left(t_{0}^{*}\right)} e^{\lambda\left(t_{0}^{*}-\rho_{i}\left(t_{0}^{*}\right)\right)} x_{i}\left(t_{0}^{*}-\rho_{i}\left(t_{0}^{*}\right)\right) \\
& +k_{i} e^{\lambda d_{k}\left(t_{0}^{*}\right)} e^{\lambda\left(t_{0}^{*}-d_{k}\left(t_{0}^{*}\right)\right)} x_{i}\left(t_{0}^{*}-d_{k}\left(t_{0}^{*}\right)\right)+e^{\lambda t_{0}^{*}} \sum_{j=1}^{n}\left|b_{i j}^{(1)}\right| L_{j}^{g}\left|y_{j}\left(t_{0}^{*}\right)\right| \\
& +e^{\lambda \tau_{i j}\left(t_{0}^{*}\right)} \sum_{j=1}^{n}\left|b_{i j}^{(2)}\right| L_{j}^{g} e^{\lambda\left(t_{0}^{*}-\tau_{i j}\left(t_{0}^{*}\right)\right)} \mid y_{j}\left(t_{0}^{*}-\tau_{i j}\left(t_{0}^{*}\right) \mid\right. \\
& \leq-\left(a_{i}-\lambda\right) K \xi_{i}+\left(a_{i}-\lambda\right) a_{i} \bar{\rho}_{i} \frac{K \xi_{i}}{1-a_{i} \bar{\rho}_{i}}+\left[a_{i} e^{\lambda \rho_{i}\left(t_{0}^{*}\right)}-a_{i}\left(1-\dot{\rho}_{i}\left(t_{0}^{*}\right)\right)\right] \frac{K \xi_{i}}{1-a_{i} \bar{\rho}_{i}} \\
& +k_{i} e^{\lambda L} \frac{K \xi_{i}}{1-a_{i} \bar{\rho}_{i}}+\sum_{j=1}^{n}\left|b_{i j}^{(1)}\right| L_{j}^{g} \frac{K \eta_{j}}{1-c_{j} \bar{r}_{j}}+e^{\lambda \bar{\tau}_{i j}} \sum_{j=1}^{n}\left|b_{i j}^{(2)}\right| L_{j}^{g} \frac{K \eta_{j}}{1-c_{j} \bar{r}_{j}} \\
& \leq\left\{-\left[\left(a_{i}-\lambda\right)\left(1-2 a_{i} \bar{\rho}_{i}\right)-a_{i}\left(e^{\lambda \bar{\rho}_{i}}-\left(1-\rho^{*}\right)\right)-k_{i} e^{\lambda L}\right] \frac{\xi_{i}}{1-a_{i} \bar{\rho}_{i}}\right. \\
& \left.+\left[\sum_{j=1}^{n}\left|b_{i j}^{(1)}\right|+e^{\lambda \bar{\tau}_{i j}} \sum_{j=1}^{n}\left|b_{i j}^{(2)}\right|\right] L_{j}^{g} \frac{\eta_{j}}{1-c_{j} \bar{r}_{j}}\right\} K=\Phi_{i}(\lambda) K<0,
\end{aligned}
$$

which contradicts (a).

If (b) holds, we get

$$
\begin{aligned}
& \dot{X}_{i}\left(t_{0}^{*}\right) \geq\left(a_{i}-\lambda\right) K \xi_{i}-\left(a_{i}-\lambda\right) a_{i} \bar{\rho}_{i} \frac{K \xi_{i}}{1-a_{i} \bar{\rho}_{i}} \\
& -\left[a_{i}-a_{i}\left(1-\dot{\rho}_{i}\left(t_{0}^{*}\right)\right) e^{-\lambda \rho_{i}\left(t_{0}^{*}\right)}\right] e^{\lambda \rho_{i}\left(t_{0}^{*}\right)} e^{\lambda\left(t_{0}^{*}-\rho_{i}\left(t_{0}^{*}\right)\right)} x_{i}\left(t_{0}^{*}-\rho_{i}\left(t_{0}^{*}\right)\right) \\
& -k_{i} e^{\lambda L} \frac{K \xi_{i}}{1-a_{i} \bar{\rho}_{i}}-e^{\lambda t_{0}^{*}} \sum_{j=1}^{n}\left|b_{i j}^{(1)}\right| L_{j}^{g}\left|y_{j}\left(t_{0}^{*}\right)\right| \\
& -e^{\lambda \tau_{i j}\left(t_{0}^{*}\right)} \sum_{j=1}^{n}\left|b_{i j}^{(2)}\right| L_{j}^{g} e^{\lambda\left(t_{0}^{*}-\tau_{i j}\left(t_{0}^{*}\right)\right)} \mid y_{j}\left(t_{0}^{*}-\tau_{i j}\left(t_{0}^{*}\right) \mid\right. \\
& \geq\left(a_{i}-\lambda\right) K \xi_{i}-\left(a_{i}-\lambda\right) a_{i} \bar{\rho}_{i} \frac{K \xi_{i}}{1-a_{i} \bar{\rho}_{i}}-\left[a_{i} e^{\lambda \rho_{i}\left(t_{0}^{*}\right)}-a_{i}\left(1-\dot{\rho}_{i}\left(t_{0}^{*}\right)\right)\right] \frac{K \xi_{i}}{1-a_{i} \bar{\rho}_{i}} \\
& -k_{i} e^{\lambda L} \frac{K \xi_{i}}{1-a_{i} \bar{\rho}_{i}}-\sum_{j=1}^{n}\left|b_{i j}^{(1)}\right| L_{j}^{g} \frac{K \eta_{j}}{1-c_{j} \bar{r}_{j}}-e^{\lambda \bar{\tau}_{i j}} \sum_{j=1}^{n}\left|b_{i j}^{(2)}\right| L_{j}^{g} \frac{K \eta_{j}}{1-c_{j} \bar{r}_{j}} \\
& \geq\left\{-\left[\left(a_{i}-\lambda\right)\left(1-2 a_{i} \bar{\rho}_{i}\right)-a_{i}\left(e^{\lambda \bar{\rho}_{i}}-\left(1-\rho^{*}\right)\right)-k_{i} e^{\lambda L}\right] \frac{\xi_{i}}{1-a_{i} \bar{\rho}_{i}}\right. \\
& \left.+\left[\sum_{j=1}^{n}\left|b_{i j}^{(1)}\right|+e^{\lambda \bar{\tau}_{i j}} \sum_{j=1}^{n}\left|b_{i j}^{(2)}\right|\right] L_{j}^{g} \frac{\eta_{j}}{1-c_{j} \bar{r}_{j}}\right\}(-K)=-\Phi_{i}(\lambda) K>0 .
\end{aligned}
$$

This is a contradiction with (b). 
Similarly, if (c) or (d) holds, we can also derive contradictory results with respect to (c) or (d), respectively. So (9) is correct. From (7) and (9), we have

$$
\left\{\begin{array}{l}
\left|X_{i}(t)\right| \leq M<K \xi_{i}, \\
\left|Y_{i}(t)\right| \leq M<K \eta_{i},
\end{array} \quad \text { for all } t \in\left(-\infty, t_{1}\right) .\right.
$$

Next, we will prove

$$
\left\{\begin{array}{l}
\left|X_{i}(t)\right| \leq M<K \xi_{i}, \\
\left|Y_{i}(t)\right| \leq M<K \eta_{i},
\end{array} \quad \text { for } t \in\left[t_{1}, t_{2}\right), i \in \widetilde{N}\right.
$$

If it is not like this, there exist $i \in \tilde{N}, t_{1}^{*} \in\left[t_{1}, t_{2}\right)$ such that one of the following cases occurs:

$$
\left\{\begin{array}{lll}
\text { (a) } X_{i}\left(t_{1}^{*}\right)=K \xi_{i}, \quad \dot{X}_{i}\left(t_{1}^{*}\right) \geq 0, & \left|X_{j}(t)\right|<K \xi_{j}, & \left|Y_{j}(t)\right|<K \eta_{j}, \\
\text { for } t \in\left(-\infty, t_{1}^{*}\right), j \in \widetilde{N}, & & \\
\text { (b) } X_{i}\left(t_{1}^{*}\right)=-K \xi_{i}, \quad \dot{X}_{i}\left(t_{1}^{*}\right) \leq 0, & \left|X_{j}(t)\right|<K \xi_{j}, & \left|Y_{j}(t)\right|<K \eta_{j}, \\
\text { for } t \in\left(-\infty, t_{1}^{*}\right), j \in \tilde{N}, & & \\
\text { (c) } Y_{i}\left(t_{1}^{*}\right)=K \eta_{i}, \quad \dot{Y}_{i}\left(t_{1}^{*}\right) \geq 0, & \left|X_{j}(t)\right|<K \xi_{j}, & \left|Y_{j}(t)\right|<K \eta_{j}, \\
\text { for } t \in\left(-\infty, t_{1}^{*}\right), j \in \widetilde{N}, & & \\
\text { (d) } Y_{i}\left(t_{1}^{*}\right)=-K \eta_{i}, \quad \dot{Y}_{i}\left(t_{1}^{*}\right) \leq 0, & \left|X_{j}(t)\right|<K \xi_{j}, & \left|Y_{j}(t)\right|<K \eta_{j}, \\
\text { for } t \in\left(-\infty, t_{1}^{*}\right), j \in \widetilde{N} . &
\end{array}\right.
$$

Similar to the proof of (9), we can deduce that (13) holds. Combining (9) and (13), we have

$$
\left\{\begin{array}{l}
\left|X_{i}(t)\right| \leq M<K \xi_{i}, \\
\left|Y_{i}(t)\right| \leq M<K \eta_{i},
\end{array} \quad \text { for all } t \in\left(-\infty, t_{2}\right) .\right.
$$

Using mathematical induction, the inequalities (8) hold. By a similar proof to (11), we have $e^{\lambda t}\left|x_{i}(t)\right| \leq \frac{K \xi_{i}}{1-a_{i} \bar{\rho}_{i}}, e^{\lambda t}\left|y_{i}(t)\right| \leq \frac{K \eta_{i}}{1-c_{i} \bar{r}_{i}}$, for $t>0$, which implies $\left|x_{i}(t)\right|=O\left(e^{-\lambda t}\right),\left|y_{i}(t)\right|=$ $O\left(e^{-\lambda t}\right), i \in \tilde{N}$. This completes the proof.

Remark 2 If the leakage delays in (3) are constant, that is, $\rho_{i}(t)=\rho, r_{i}(t)=r$. Assumption 2 is changed into the following form.

Assumption 2' Let $a_{i} \rho<1, c_{i} r<1$, for all $i \in \tilde{N}$. There exist positive constants $\xi_{1}, \xi_{2}, \ldots, \xi_{n}$ and $\eta_{1}, \eta_{2}, \ldots, \eta_{n}$ such that, for $t>0$ and $i \in \widetilde{N}$, the following conditions hold:

$$
\left\{\begin{array}{l}
-\left[a_{i}\left(1-2 \rho a_{i}\right)-k_{i}\right] \frac{1}{1-a_{i} \rho} \xi_{i}+\left[\sum_{j=1}^{n}\left|b_{i j}^{(1)}\right|+\sum_{j=1}^{n}\left|b_{i j}^{(2)}\right|\right] L_{j}^{g} \frac{1}{1-c_{j} r} \eta_{j}<0 \\
-\left[c_{i}\left(1-2 r c_{i}\right)-l_{i}\right] \frac{1}{1-c_{i} r} \eta_{i}+\left[\sum_{j=1}^{n}\left|d_{i j}^{(1)}\right|+\sum_{j=1}^{n}\left|d_{i j}^{(2)}\right|\right] L_{j}^{f} \frac{1}{1-a_{j} \rho} \xi_{j}<0 .
\end{array}\right.
$$

Similar to the proof of Theorem 1, we get the following result.

Corollary 1 If Assumptions 1 and 2' hold, the BAM neural networks with constant leakage delays and the sampled-data state feedback inputs are exponentially stable. 


\section{Simulation example}

In this section, we give an illustrative example to show the efficiency of our theoretical results.

Example 1 Consider the following BAM neural network with leakage delays and sampleddata state feedback inputs:

$$
\left\{\begin{array}{l}
\dot{x}(t)=-A x\left(t-\rho_{i}(t)\right)+B_{1} g(y(t))+B_{2} g\left(y(t-\tau(t))-K x\left(t_{k}\right),\right. \\
\dot{y}(t)=-C y\left(t-r_{i}(t)\right)+D_{1} f(x(t))+D_{2} f\left(x(t-\sigma(t))-L y\left(t_{k}\right),\right.
\end{array}\right.
$$

where

$$
\begin{array}{ll}
A=\left[\begin{array}{lll}
1 & 0 & 0 \\
0 & 1 & 0 \\
0 & 0 & 1
\end{array}\right], & C=\left[\begin{array}{ccc}
0.9 & 0 & 0 \\
0 & 0.9 & 0 \\
0 & 0 & 0.9
\end{array}\right], \\
B_{1}=\left[\begin{array}{ccc}
0 & 0 & -0.2 \\
-0.2 & 0 & 0.5 \\
0 & 0.2 & 1
\end{array}\right], & B_{2}=\left[\begin{array}{ccc}
1 & 0 & 0.2 \\
-0.2 & 0 & 0.4 \\
0.2 & -0.2 & 0
\end{array}\right], \\
D_{1}=\left[\begin{array}{ccc}
0.1 & -0.1 & 0 \\
-0.1 & 0.1 & 0.1 \\
0 & 0 & 0.1
\end{array}\right], & D_{2}=\left[\begin{array}{ccc}
0.1 & 0 & 0 \\
0 & 0.1 & 0 \\
0 & 0 & 0.1
\end{array}\right],
\end{array}
$$

and the sampled-data gain

$$
K=L=\left[\begin{array}{ccc}
0.2 & 0 & 0 \\
0 & 0.2 & 0 \\
0 & 0 & 0.2
\end{array}\right]
$$

The activation functions are taken as $f(\cdot)=g(\cdot)=0.4 \tanh (\cdot)$. Time-varying delays are chosen as $\tau(t)=0.1|\sin t|, \sigma(t)=0.1|\cos t|$ and the leakage delays are chosen as $\rho_{i}(t)=$ $0.2+0.01 \sin t, r_{i}(t)=0.2+0.01 \cos t$, respectively.

It is easy to verify $a_{i} \bar{\rho}_{i}<1, c_{i} \bar{r}_{i}<1$. Select $\xi_{i}=20, \eta_{i}=10, i=1,2,3$, and we obtain

$$
\left\{\begin{array}{l}
-\left[a_{1}\left(1-2 a_{1} \bar{\rho}_{1}\right)-a_{1} \rho_{1}^{*}-k_{1}\right] \frac{1}{1-a_{1} \bar{\rho}_{1}} \xi_{1}+\left[\sum_{j=1}^{3}\left|b_{1 j}^{(1)}\right|+\sum_{j=1}^{3}\left|b_{1 j}^{(2)}\right|\right] L_{1 \frac{1}{1-c_{1} \bar{r}_{1}}}^{g} \eta_{1} \\
\quad=-2.9207<0, \\
-\left[a_{2}\left(1-2 a_{2} \bar{\rho}_{2}\right)-a_{2} \rho_{2}^{*}-k_{2}\right] \frac{1}{1-a_{2} \bar{\rho}_{2}} \xi_{2}+\left[\sum_{j=1}^{3}\left|b_{2 j}^{(1)}\right|+\sum_{j=1}^{3}\left|b_{2 j}^{(2)}\right|\right] L_{2}^{g} \frac{1}{1-c_{2} \bar{r}_{2}} \eta_{2} \\
\quad=-3.4085<0, \\
-\left[a_{3}\left(1-2 a_{3} \bar{\rho}_{3}\right)-a_{3} \rho_{3}^{*}-k_{3}\right] \frac{1}{1-a_{3} \bar{\rho}_{3}} \xi_{3}+\left[\sum_{j=1}^{3}\left|b_{3 j}^{(1)}\right|+\sum_{j=1}^{3}\left|b_{3 j}^{(2)}\right|\right] L_{3}^{g} \frac{1}{1-c_{3} \bar{r}_{3}} \eta_{3} \\
\quad=-1.9451<0, \\
-\left[c_{1}\left(1-2 c_{1} \bar{r}_{1}\right)-c_{1} r_{1}^{*}-l_{1}\right] \frac{1}{1-c_{1} \bar{r}_{1}} \eta_{1}+\left[\sum_{j=1}^{3}\left|d_{1 j}^{(1)}\right|+\sum_{j=1}^{3}\left|d_{1 j}^{(2)}\right|\right] L_{1}^{f} \frac{1}{1-a_{1} \bar{\rho}_{1}} \xi_{1} \\
\quad=-1.4756<0, \\
-\left[c_{2}\left(1-2 c_{2} \bar{r}_{2}\right)-c_{2} r_{2}^{*}-l_{2}\right] \frac{1}{1-c_{2} \bar{r}_{2}} \eta_{2}+\left[\sum_{j=1}^{3}\left|d_{2 j}^{(1)}\right|+\sum_{j=1}^{3}\left|d_{2 j}^{(2)}\right|\right] L_{2}^{f} \frac{1}{1-a_{2} \bar{\rho}_{2}} \xi_{2} \\
=-0.4756<0, \\
-\left[c_{3}\left(1-2 c_{3} \bar{r}_{3}\right)-c_{3} r_{3}^{*}-l_{3}\right] \frac{1}{1-c_{3} \bar{r}_{3}} \eta_{3}+\left[\sum_{j=1}^{3}\left|d_{3 j}^{(1)}\right|+\sum_{j=1}^{3}\left|d_{3 j}^{(2)}\right|\right] L_{3}^{f} \frac{1}{1-a_{3} \bar{\rho}_{3}} \xi_{3} \\
\quad=-0.4756<0 .
\end{array}\right.
$$



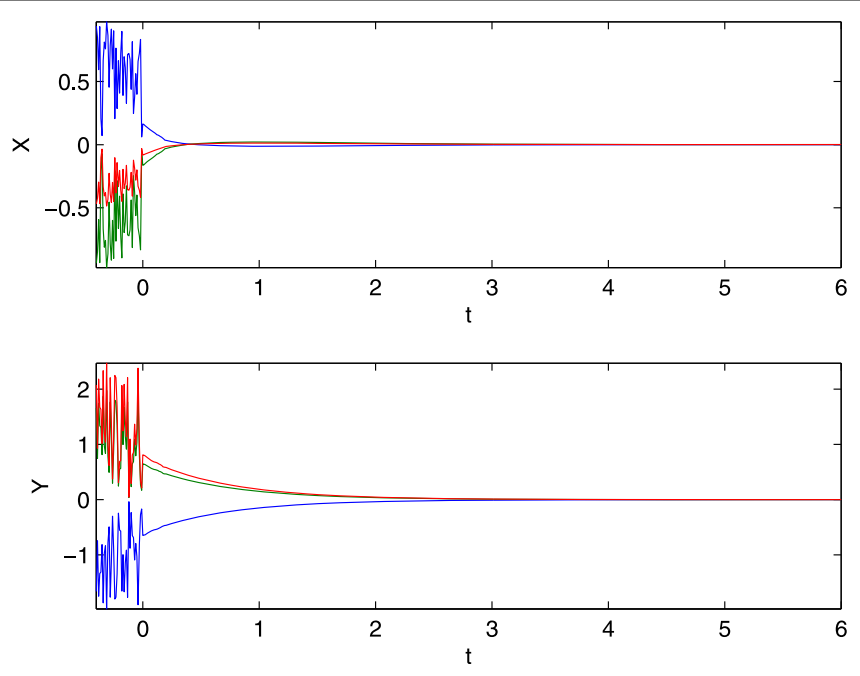

Figure 1 State trajectory of the system [17].

This means that all conditions in Theorem 1 are satisfied. Hence, by Theorem 1 (17) is exponentially stable. On the other hand, we have the following simulation result, shown in Figure 1.

\section{Conclusion}

In this paper, we investigate the stability of BAM neural networks with leakage delays and a sampled-data input. By using the time-delay approach, the conditions for ensuring the exponential stability of the system are derived. It should be pointed out that there are many papers focusing on the stability problem of sampled-data systems, leakage delay, and sampled-data state feedback that have never been taken into consideration in the BAM neural networks. To the best of our knowledge, this is the first time to consider the stability of BAM neural networks with both leakage delays and sampled-data state feedback at the same time. The results of this paper are worthy as complementary to the existing results. Finally, a numerical example and its computer simulations have been presented to show the effectiveness of our theoretical results.

Competing interests

The authors declare that they have no competing interests.

Authors' contributions

LL carried out the main results of this paper and drafted the manuscript. YY directed the study and helped to inspect the manuscript. All authors read and approved the final manuscript.

\section{Author details}

'School of Science, Jiangnan University, Wuxi, 214122, China. ${ }^{2}$ Key Laboratory of Advanced Process Control for Light Industry (Ministry of Education), Jiangnan University, Wuxi, 214122, China.

\section{Acknowledgements}

This work was jointly supported by the National Natural Science Foundation of China under Grant 60875036, the Foundation of Key Laboratory of Advanced Process Control for Light Industry (Jiangnan university), Ministry of Education, P.R. China the Fundamental Research Funds for the Central Universities (JUSRP51317B, JUDCF13042).

Received: 31 October 2013 Accepted: 6 January 2014 Published: 27 Jan 2014

\section{References}

1. Kosko, B: Adaptive bi-directional associative memories. Appl. Opt. 26, 4947-4960 (1987) 
2. Kosko, B: Bi-directional associative memories. IEEE Trans. Syst. Man Cybern. 18, 49-60 (1988)

3. Gao, M, Cui, B: Global robust exponential stability of discrete-time interval BAM neural networks with time-varying delays. Appl. Math. Model. 33(3), 1270-1284 (2009)

4. Ailong, W, Zhigang, Z: Dynamic behaviors of memristor-based recurrent neural networks with time-varying delays. Neural Netw. 36, 1-10 (2012)

5. Wang, Z, Zhang, H: Global asymptotic stability of reaction-diffusion Cohen-Grossberg neural networks with continuously distributed delays. IEEE Trans. Neural Netw. 21(1), 39-48 (2010)

6. Li, Y, Yang, C: Global exponential stability analysis on impulsive BAM neural networks with distributed delays. J. Math. Anal. Appl. 324(2), 1125-1139 (2006)

7. Lou, X, Ye, Q, Cui, B: Exponential stability of genetic regulatory networks with random delays. Neurocomputing 73 , 759-769 (2010)

8. Gopalsamy, K: Leakage delays in BAM. J. Math. Anal. Appl. 325, 1117-1132 (2007)

9. Zhang, $\mathrm{H}$, Shao, J: Existence and exponential stability of almost periodic solutions for CNNs with time-varying leakage delays. Neurocomputing 74, 226-233 (2013)

10. Arik, S, Tavsanoglu, V: Global asymptotic stability analysis of bidirectional associative memory neural networks with constant time delays. Neurocomputing 68, 161-176 (2005)

11. Cao, J, Wang, L: Periodic oscillatory solution of bidirectional associative memory networks with delays. Phys. Rev. E 61 $1825-1828$ (2000)

12. Liu, Z, Chen, A, Cao, J: Existence and global exponential stability of almost periodic solutions of BAM neural networks with distributed delays. Phys. Lett. A 319, 305-316 (2003)

13. Chen, A, Huang, L, Cao, J: Existence and stability of almost periodic solution for BAM neural networks with delays. Appl. Math. Comput. 137, 177-193 (2003)

14. Liu, B: Global exponential stability for BAM neural networks with time-varying delays in the leakage terms. Nonlinear Anal., Real World Appl. 14, 559-566 (2013)

15. Peng, S: Global attractive periodic solutions of BAM neural networks with continuously distributed delays in the leakage terms. Nonlinear Anal., Real World Appl. 11, 2141-2151 (2010)

16. Balasubramaniam, P, Kalpana, M, Rakkiyappan, R: Global asymptotic stability of BAM fuzzy cellular neural networks with time delay in the leakage term, discrete and unbounded distributed delays. Math. Comput. Model. 53, 839-853 (2011)

17. Lakshmanan, S, Park, J, Lee, T, Jung, H, Rakkiyappan, R: Stability criteria for BAM neural networks with leakage delays and probabilistic time-varying delays. Appl. Math. Comput. 219, 9408-9423 (2013)

18. Wu, Z, Shi, P, Su, H, Chu, J: Sampled-data synchronization of chaotic Lur'e systems with time delays. IEEE Trans. Neural Netw. Learn. Syst. 24(3), 410-421 (2013)

19. Gan, Q: Synchronisation of chaotic neural networks with unknown parameters and random time-varying delays based on adaptive sampled-data control and parameter identification. IET Control Theory Appl. 6(10), 1508-1515 (2012)

20. Lee, T, Park, J, Kwon, O, Lee, S: Stochastic sampled-data control for state estimation of time-varying delayed neural networks. Neural Netw. 46, 99-108 (2013)

21. Hu, J, Li, N, Liu, X, Zhang, G: Sampled-data state estimation for delayed neural networks with Markovian jumping parameters. Nonlinear Dyn. 73, 275-284 (2013)

22. Rakkiyappan, R, Sakthivel, N, Park, J, Kwon, O: Sampled-data state estimation for Markovian jumping fuzzy cellular neural networks with mode-dependent probabilistic time-varying delays. Appl. Math. Comput. 221, 741-769 (2013)

23. Fridman, E, Blighovsky, A: Robust sampled-data control of a class of semilinear parabolic systems. Automatica 48, 826-836 (2012)

24. Samadi, B, Rodrigues, L: Stability of sampled-data piecewise affine systems: a time-delay approach. Automatica 45, 1995-2001 (2009)

25. Rodrigues, L: Stability of sampled-data piecewise-affine systems under state feedback. Automatica 43, $1249-1256$ (2007)

26. Zhang, C, Jiang, L, He, Y, Wu, H, Wu, M: Stability analysis for control systems with aperiodically sampled data using an augmented Lyapunov functional method. IET Control Theory Appl. 7(9), 1219-1226 (2013)

27. Seuret, A, Peet, M: Stability analysis of sampled-data systems using sum of squares. IEEE Trans. Autom. Control 58(6), 1620-1625 (2013)

28. Oishi, Y, Fujioka, H: Stability and stabilization of aperiodic sampled-data control systems using robust linear matrix inequalities. Automatica 46, 1327-1333 (2010)

29. Feng, L, Song, Y: Stability condition for sampled data based control of linear continuous switched systems. Syst. Control Lett. 60, 787-797 (2011)

10.1186/1687-1847-2014-39

Cite this article as: Li et al.: The exponential stability of BAM neural networks with leakage time-varying delays and sampled-data state feedback input. Advances in Difference Equations 2014, 2014:39 